Case Reports in
Gastroenterology
Case Rep Gastroenterol 2020;14:448-452

DOI: 10.1159/000508426

Published online: August 26, 2020

(C) 2020 The Author(s)

Published by S. Karger AG, Base

www.karger.com/crg

This article is licensed under the Creative Commons Attribution-NonCommercial 4.0 International License (CC BY-NC) (http://www.karger.com/Services/OpenAccessLicense).

Usage and distribution for commercial purposes requires written permission.

\title{
Alcoholic Hepatitis: A Common Disease with Uncommon Presentation
}

\author{
Pratik Khatiwada $^{\mathrm{a}}$ Jose A. Rodriguez ${ }^{\mathrm{a}}$ Andres Reyes-Corcho ${ }^{\mathrm{a}}$ \\ aDepartment of Internal Medicine, Memorial Healthcare System, Pembroke Pines, FL, USA
}

\section{Keywords}

Alcoholic hepatitis $\cdot$ Hyperbilirubinemia $\cdot$ Hypertriglyceridemia

\begin{abstract}
Alcoholic liver disease is one of the leading causes of hepatitis, cirrhosis, liver failure, and hepatic carcinoma. Alcoholic hepatitis refers to the acute onset of symptomatic hepatitis. We describe a patient who presented with alcoholic hepatitis with direct hyperbilirubinemia, elevated alkaline phosphatase (ALP), and hypertriglyceridemia. All the imaging and laboratory work excluded obstructive causes, and liver biopsy confirmed the diagnosis. Alcoholic hepatitis is a common condition, but some unusual presentations can lead one to think of uncommon pathologies. This case reflects that alcoholic hepatitis can present with high levels of triglycerides as well as a cholestatic pattern with direct hyperbilirubinemia and ALP without an obvious obstruction cause.

(C) 2020 The Author(s)

Published by S. Karger AG, Basel
\end{abstract}

\section{Introduction}

Alcoholic liver disease (ALD) refers to a spectrum of dysplastic and inflammatory changes to hepatic tissues including steatosis, inflammation, fibrosis, and cirrhosis, which are typically co-occurring phenomena resulting from chronic excessive alcohol consumption [1]. Alcoholic hepatitis refers to the acute onset of symptomatic hepatitis with symptoms such as right upper quadrant pain, jaundice, anorexia, fever, fatigue, and tender hepatomegaly. The diagnosis 


\section{Case Reports in Gastroenterology}

Case Rep Gastroenterol 2020;14:448-452

DOI: $10.1159 / 000508426$

(c) 2020 The Author(s). Published by S. Karger AG, Basel www.karger.com/crg

Khatiwada et al.: Alcoholic Hepatitis: A Common Disease with Uncommon Presentation

of ALD is made clinically based on a history of significant alcohol intake and supportive laboratory findings such as elevated liver enzymes AST, ALT( with AST/ALT ratio >2), GGT, and alkaline phosphatase (ALP) along with elevated bilirubin coupled with peripheral stigmata of liver disease and rarely requires a liver biopsy [2,3]. Usually, ALD presents with indirect hyperbilirubinemia, but we present a rare case of ALD with direct hyperbilirubinemia, elevated ALP, and hypertriglyceridemia. The patient underwent extensive laboratory and imaging tests that excluded extrahepatic cholestasis and viral, autoimmune, toxic, and ischemic hepatitis. Liver biopsy ruled out nonalcoholic etiologies of such a rare pattern.

\section{Case Presentation}

A 27-year-old Hispanic male was admitted from the emergency department with a history of progressive worsening yellowish discoloration of the skin and eyes, nausea, and fatigue for the last 7 days. It was associated with generalized pruritus. He denied abdominal pain, fever, chills, diarrhea, abdominal distention, melena, hematemesis, myalgia, joint pain, and other symptoms at the time of presentation. The patient recollected a similar episode of yellowish discoloration about a year ago, but it resolved without medical attention. He was not taking any medications. He denied a family history of jaundice and liver disorders on his mother's side. The patient lived with his mother and worked at a veterinary hospital as an animal handler. He had a history of EtOH consumption since the age of 14 and mentioned that until last year he was consuming 1/2 L of Vodka daily, but due to gastric reflux issues he switched to beer. He admitted drinking about 4 beers per day, reporting multiple failed attempts at quitting. He denied intravenous drug use, blood transfusion, and multiple sexual partners. Physical examination was notable for icteric sclera and jaundice of his whole body including the palms and soles. Abdominal examination revealed marked hepatomegaly with liver edge palpable up to $5 \mathrm{~cm}$ below the right costal margin. There were no signs of peripheral stigmata of liver disease, and his BMI was 24.

Initial laboratory studies were significant for elevated liver enzymes (AST $170 \mathrm{IU} / \mathrm{L}$, ALT $64 \mathrm{IU} / \mathrm{L}$, ALP $649 \mathrm{IU} / \mathrm{L}$, GGT 3,792 U/L), elevated total bilirubin of $15.7 \mathrm{mg} / \mathrm{dL}$ with direct bilirubin of $11.75 \mathrm{mg} / \mathrm{dL}$, albumin $2.2 \mathrm{mg} / \mathrm{dL}$, and a blood EtOH level of 399. Maddrey's discriminant function was calculated to be 14.8. The rest of the laboratory findings were within normal limits including BMP, ammonia, PT, INR, and platelet counts. Urine toxicology screen was negative for drugs and toxins. The patient was started on intravenous fluids with normal saline, intravenous thiamine, and folic acid.

Upon admission, the presumptive diagnosis was choledocholithiasis presenting with an extrahepatic cholestatic pattern of liver enzymes and hyperbilirubinemia versus intrahepatic cholestasis (viral hepatitis, ALD). Abdominal sonogram showed hepatomegaly, diffused fatty infiltration of liver, nonspecific gallbladder distention, and gallbladder wall thickening/edema. Abdominal imaging including MRI/MRCP ruled out CBD stones and biliary duct dilatation but revealed hepatic steatosis, pancreatitis, and distended gallbladder wall with mild edematous wall. Acute and chronic viral hepatitis serologies (HAV, HBV, HCV) were negative. ERCP was not performed after MRCP ruled out CBD obstruction.

Due to elevated direct bilirubin, alternative etiologies such as primary biliary cirrhosis, primary sclerosing cholangitis, Wilson's disease, and hemochromatosis were also considered as co-existing pathologies, and further workup was continued while the patient was being managed with intravenous fluids, benzodiazepines, and alcohol cessation. Other laboratory results included borderline elevated lipase and ceruloplasmin, anemia with markedly 


\section{Case Reports in Gastroenterology}

Case Rep Gastroenterol 2020;14:448-452 DOI: $10.1159 / 000508426$

(c) 2020 The Author(s). Published by S. Karger AG, Basel www.karger.com/crg

Khatiwada et al.: Alcoholic Hepatitis: A Common Disease with Uncommon Presentation

increased RDW, increased circulating nucleated RBCs, and macrocytic target cells. ANA screen and antimitochondrial antibody were negative. HIV-1/2 Ag/Ab was negative. Cholesterol (483 mg/dL), LDL (362 mg/dL), and triglycerides $(1,040 \mathrm{mg} / \mathrm{dL}$ ) were markedly increased with decreased HDL (12 mg/dL). Electrophoretic HbA1c was normal, and abnormal hemoglobin was not identified. He had an elevated ferritin level of 1,693.6 ng/mL. Hereditary hemochromatosis DNA mutational analysis was negative for C282Y and H63D mutations in the HFE gene. Antinuclear antibody, anti-mitochondrial antibody, ANCA screen, PR-3 antibody, and MPO antibody tests were negative.

Ultrasound-guided core needle liver biopsy was performed 5 days after presentation which showed diffuse steatosis ( $>70 \%)$, primarily macrovesicular, with scattered lobular and portal inflammation, and hepatocyte ballooning degeneration - findings consistent with alcohol-related etiology (Fig. 1a-d).

Clinically, his symptoms were better during the hospital stay; liver enzymes and bilirubin trended up for 2 days before trending down. He was discharged after 6 days of conservative treatment with alcohol cessation counseling and recommendations to follow up in the gastroenterology office.

\section{Discussion}

ALD is clinically silent with little or no symptoms, especially in the patients with early ALD and compensated cirrhosis. On top of that, most patients are reluctant to openly admit their drinking behavior, so diagnosis depends highly on clinical suspicion, various laboratory tests, and sometimes even invasive tests.

In most cases, ALD can be diagnosed easily with reliable history, hepatic function panel, and imaging to support the diagnosis [4]. Patients with ALD typically have elevated red blood cell MCV, moderate elevations of AST and ALT (typically less than $400 \mathrm{IU} / \mathrm{L}$ with AST/ALT ratio of $>2$ ), elevated serum bilirubin, GGT, and INR, and low albumin and platelet count [5]. Acute-phase reactants such as serum ceruloplasmin, ferritin, and alpha- 1 antitrypsin may be elevated in patients with severe alcoholic hepatitis.

Generally, clinical and laboratory features are often adequate to establish the diagnosis of ALD in a patient with long-standing history of heavy alcohol use. In our patient with his AST/ALT ratio of 170/64 (=2.65), elevated serum bilirubin, low albumin $(2.2 \mathrm{mg} / \mathrm{dL})$, and ruling out other common causes of acute hepatitis, alcoholic hepatitis was the most likely diagnosis. However, the unusual cholestatic pattern of elevated direct bilirubin and markedly elevated ALP (649 IU/L) and high ferritin level $(1,693.6 \mathrm{ng} / \mathrm{mL})$ added more to the diagnostic dilemma in our case.

While many patients with suspected alcoholic hepatitis will not require a liver biopsy, we ended up ordering an ultrasound-guided liver biopsy in our patient because of the questionable differential diagnosis and unknown family history of liver disease on the father's side. The biopsy demonstrated toxic hepatocellular injury most supportive of alcohol etiology and ruled out any evidence of iron overload to suggest primary (hemochromatosis) or secondary hemosiderosis or alpha- 1 antitrypsin deficiency. Biopsy also ruled out significant biliary duct obstruction.

Painless jaundice with elevated direct bilirubin and elevated ALP is commonly attributed to choledocholithiasis or hepatobiliary, pancreatic carcinoma. ALD can also manifest with clinical and histological evidence of cholestasis with elevated ALP but generally up to 2-3 times 
the upper limit of normal [6]. This case represents a rare manifestation of ALD with marked elevation of direct bilirubin and ALP.

Alcoholic hepatitis is a common condition, but some presentations can lead one to think of unusual pathologies. This case reflects that alcoholic hepatitis can present with high levels of triglycerides as well as a cholestatic pattern with direct hyperbilirubinemia and ALP without an obvious obstruction cause.

\section{Statement of Ethics}

The patient gave written informed consent to write this case, and the protocol was approved by the institute's committee on human research.

\section{Conflict of Interest Statement}

All authors have no financial disclosure or conflicts of interest.

\section{Funding Sources}

This research was performed under the guidance of the Memorial Healthcare System Internal Medicine Residency, without specific funding support.

\section{Author Contributions}

Pratik Khatiwada and Jose A. Rodriguez: data collection, manuscript preparation, and literature search. Andres Reyes-Corcho: data interpretation and manuscript preparation.

\section{References}

1 Spengler EK, Dunkelberg J, Schey R. Alcoholic hepatitis: current management. Dig Dis Sci. 2014 Oct;59(10):2357-66.

2 Chacko KR, Reinus J. Spectrum of Alcoholic Liver Disease. Clin Liver Dis. 2016 Aug;20(3):419-27.

3 Lucey MR, Mathurin P, Morgan TR. Alcoholic hepatitis. N Engl J Med. 2009 Jun;360(26):2758-69.

4 Torruellas C. Diagnosis of alcoholic liver disease. World J Gastroenterol. 2014 Sep;20(33):116.

5 Morgan TR. Management of alcoholic hepatitis. Gastroenterol Hepatol (N Y). 2007 Feb;3(2):97-9.

6 Tung BY, Carithers RL Jr. Cholestasis and alcoholic liver disease. Clin Liver Dis. 1999 Aug;3(3):585-601. 

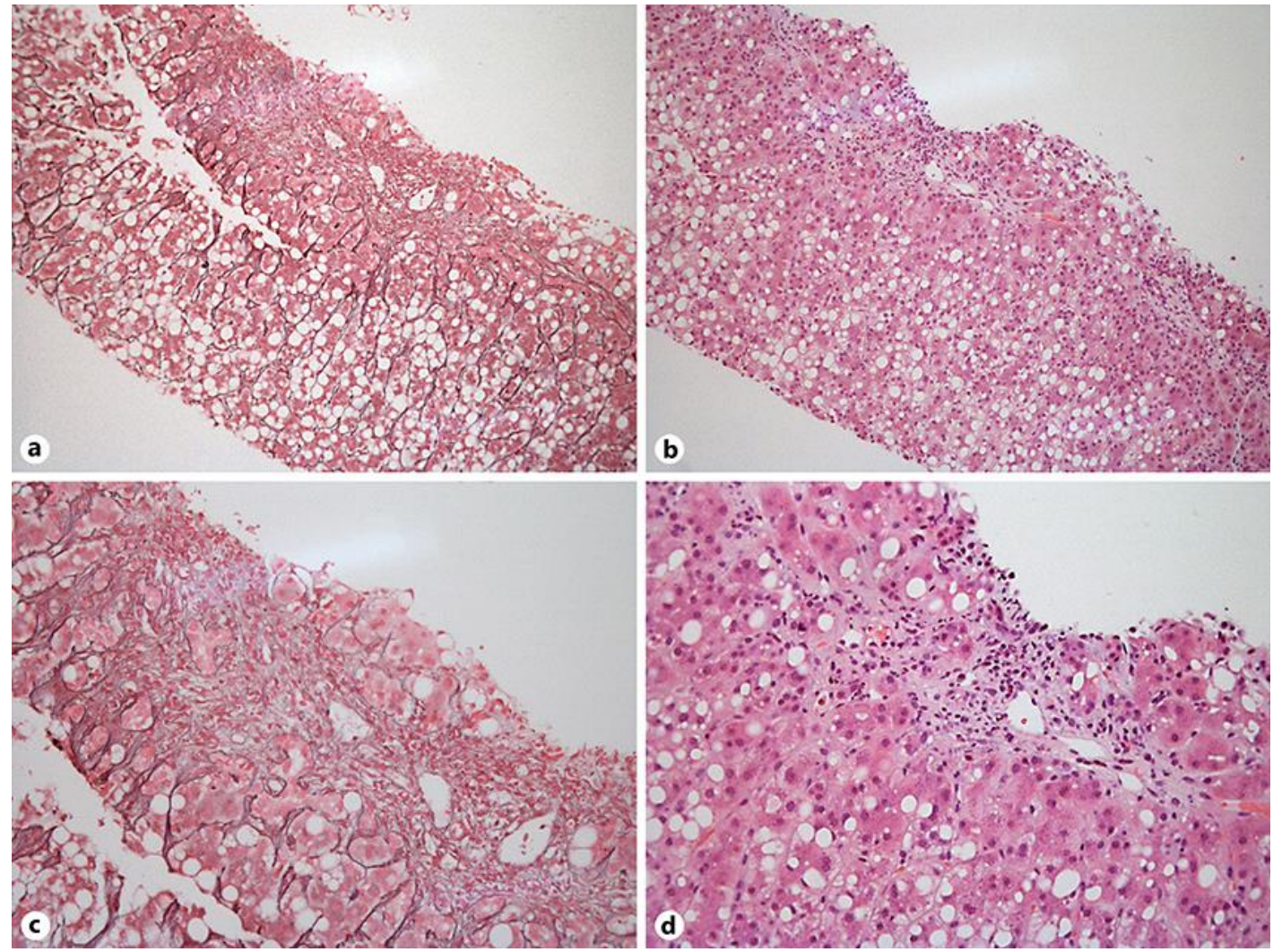

Fig. 1. a-d Liver core biopsy. H\&E stain, CK19, CK7, and Retic at both $10 \times$ and $20 \times$ magnifications. a-d Diffuse steatosis ( $>70 \%)$, primarily macrovesicular, with scattered lobular and portal inflammation, and hepatocyte ballooning degeneration. c Mallory hyaline and rare acidophilic bodies, and mild pericellular and periportal fibrosis (trichome stain). $\mathbf{d}$ No parenchymal iron deposition (iron stain); hepatocyte intracytoplasmic hyaline globules suggestive of alpha-1 antitrypsin deficiency are absent (PAS stain with diastase). 